\title{
Vortex pinning in a superconducting film due to in-plane magnetized ferromagnets of different shapes: The London approximation
}

\author{
M. V. Milošević and F. M. Peeters* \\ Departement Natuurkunde, Universiteit Antwerpen (Campus Drie Eiken), Universiteitsplein 1, B-2610 Antwerpen, Belgium
}

(Received 15 October 2003; published 24 March 2004)

\begin{abstract}
The London approach is used to study the interaction between a superconducting vortex in a type-II superconducting film (SC) and a ferromagnet (FM) with in-plane magnetization. We calculated numerically the dependence of the pinning behavior on the FM-vortex distance, SC film thickness, and geometry of the magnetic structure and its magnetization. Dual behavior of the interaction energy in terms of the field-polaritydependent pinning was found. For weak magnetization such that no vortex-antivortex pair is induced by the FM an external vortex will be pinned near the negative pole of the FM. In the presence of a vortex-antivortex pair the external vortex is pinned on the positive (negative) pole of the FM if the magnetization of the FM is below (above) some critical value. Further, we investigated the interaction of a vortex and an in-plane magnetic stripe ("wall"). Such SC-FM heterostructure is found to be useful in applications where vortex-free superconducting areas are needed. For a more complicated geometry of the FM, i.e., magnets with edge defects, we discuss the breaking of the vortex-FM interaction symmetry which results in different local minima for the energetically favorable vortex position.
\end{abstract}

DOI: 10.1103/PhysRevB.69.104522

PACS number(s): 74.78.-w, 74.25.Qt, 74.25.Ha

\section{INTRODUCTION}

Arrays of submicron size ferromagnetic particles are potential devices for prospective applications in which high current densities or high magnetic fields are involved. The possibility of enhancing the critical currents by magnetic nanostructuring has drawn a lot of attention over the last decade, especially after significant progress in modern nanolithography techniques. The rich diversity of the mixed state in the superconducting films shows that vortex pinning and dynamics are also highly interesting from a fundamental point of view.

To study the effects due to the interplay of the superconducting order parameter and the nonhomogeneous magnetic field resulting from the ferromagnet, several experimental groups fabricated periodic arrays of magnetic dots over or under the superconducting film. ${ }^{1-3}$ Such ferromagnetic dots act as very effective trapping centers for the vortices, which prevent energy dissipation and lead to an enhancement of the critical current. Bulaevskii et al. ${ }^{4}$ suggested that the pinning of vortices in superconductor (SC)/ferromagnetic (FM) multilayers can be many times larger than the pinning by columnar defects. Although numerous experiments have explored its macroscopic nature, the pinning mechanism of the vortices by the magnetic dots is still not completely understood. Other theoretical studies involving finite-size ferromagnets were mainly restricted to the problem of a magnetic dot with out-of-plane magnetization embedded in a superconducting film. 5

In the last few years, models for the interaction between a superconducting film and a ferromagnet placed on top of it appeared. ${ }^{7-9}$ In these models, due to the fact that the sizes of all structures are much larger than the coherence length $\xi$, the London theory was used. The magnetic texture interacts with the SC current, which subsequently changes the mag- netic field. The thickness of the SC film and of the FM was assumed to be extremely small (i.e., $d \ll \xi, \lambda$ ). Elementary solutions for the interaction between the out-of-plane magnetized circular magnetic dot (bubble) and annulus (ring) with a vortex were found. If extended, these models can be used for arbitrary direction of the magnetization, but even in the thin SC film approximation, complicated mathematical formalism cannot be avoided.

Most recently, the pinning of vortices by small magnetic particles was studied experimentally. ${ }^{10-12}$ In Ref. 13 we approximated the magnetic-field profile of a ferromagnet by a magnetic dipole and found analytical expressions for all relevant quantities within the London approach. In the present paper, we generalize those results to include the realistic magnetic-field profile of a FM of arbitrary shape when magnetized in the direction parallel to the SC. The superconducting film lies in the $x y$ plane while the FM is positioned at distance $l$ above the SC, and is magnetized in the positive $x$ direction (in plane). To avoid the proximity effect and the exchange of electrons between the FM and the SC we assume a thin layer of insulating oxide between them, as is usually the case in the experiment. A similar study for the less complicated case of perpendicular magnetization was presented in Ref. 14.

The paper is organized as follows. In the following section we present the general formalism. In Sec. III, we discuss the pinning potential of the magnetic stripe, a plane of inplane magnetized dipoles, perpendicular to the SC plane. Further, in Sec. IV we investigate the field-polaritydependent pinning in the case of an in-plane magnetized bar on top of a superconductor and compare our results with the experimental ones. The influence of the geometry of the magnet on the pinning of external flux lines is analyzed in Sec. V. The effect of the presence of symmetric defects in the magnet geometry is then discussed, and our conclusions are given in Sec. VI. 


\section{THEORETICAL FORMALISM}

We consider a ferromagnet of arbitrary shape with homogeneous in-plane magnetization $\vec{M}$, placed outside a type-II $\mathrm{SC}$ film interacting with a single vortex in the SC. The complete description of our approach, based on solving the London equation on conjoined half spaces, can be found in our earlier works. ${ }^{13,14}$ The direct interaction energy between the vortex and the FM in a stationary magnet-superconductor system is given by ${ }^{14}$

$$
U_{m v}=\frac{1}{2 c} \int d V^{(i)}\left[\vec{j}_{m} \cdot \vec{\Phi}_{v}\right]-\frac{1}{2} \int d V^{(f m)} \vec{h}_{v} \cdot \vec{M},
$$

where $\vec{\Phi}_{v}=\left(\Phi_{\rho}, \Phi_{\varphi}, \Phi_{z}\right)=\left(0, \Phi_{0} /(2 \pi \rho), 0\right)$ denotes the vortex magnetic flux vector $\left(\Phi_{0}\right.$ is the flux quantum). The first integration is performed over the volume inside, $V^{(i)}$, the superconductor, while $V^{(f m)}$ in the second integral denotes the volume of the ferromagnet. Indexes $v$ and $m$ refer to the vortex and the magnet, respectively, $\vec{j}$ denotes the current, and $\vec{h}$ the magnetic field. In expression (1), the integral over the remote surface ${ }^{14}$ is omitted since the magnetization is confined in a limited volume, and the field, vector potential, and current decrease sufficiently fast at infinity.

As described elsewhere, ${ }^{13,14}$ the interaction energy in this system consists of two parts: (i) the interaction between the Meissner currents generated in the SC $\left(\vec{j}_{m}\right)$ by the FM and the vortex and (ii) the interaction between the vortex magnetic field and the FM. From the analytic expressions given in Ref. 13 it can be shown that in the case of a point magnetic dipole (MD) these two contributions are equal for both in- and out-of-plane magnetization. Due to the superposition principle, the finite FM's with homogeneous magnetization can be represented as an infinite number of dipoles. Consequently, in the present case the vortex-magnet interaction energy equals

$$
U_{m v}=-\int d V^{(f m)} \vec{h}_{v} \cdot \vec{M} .
$$

In order to obtain the current induced in the superconductor by the ferromagnet, one should solve first the equation for the vector potential

$$
\operatorname{rot}\left(\operatorname{rot} \vec{A}_{m}\right)+\frac{1}{\lambda^{2}} \Theta(d / 2-|z|) \vec{A}_{m}=4 \pi \operatorname{rot} \vec{M}
$$

This equation is rather complicated to be handled for a finitesize FM. However, we need only the analytic expression for the induced SC current in an infinite superconducting film with thickness $d(-d / 2<z<d / 2)$ in the in-plane MD case (with magnetic moment $m$ ) which was obtained earlier ${ }^{13}$ as

$$
\begin{aligned}
j_{x}^{m d}(x, y, z)= & -\frac{c m \Phi_{0}}{2 \pi \lambda^{3}} \frac{y_{m}^{\prime} x_{m}^{\prime}}{R_{m}^{2}} \int_{0}^{\infty} d q \exp \left\{-q\left(\left|z_{m}\right|-\frac{d}{2}\right)\right\} \\
& \times q^{2}\left(\frac{2 J_{1}\left(q R_{m}\right)}{q R_{m}}-J_{0}\left(q R_{m}\right)\right) C(q, z)
\end{aligned}
$$

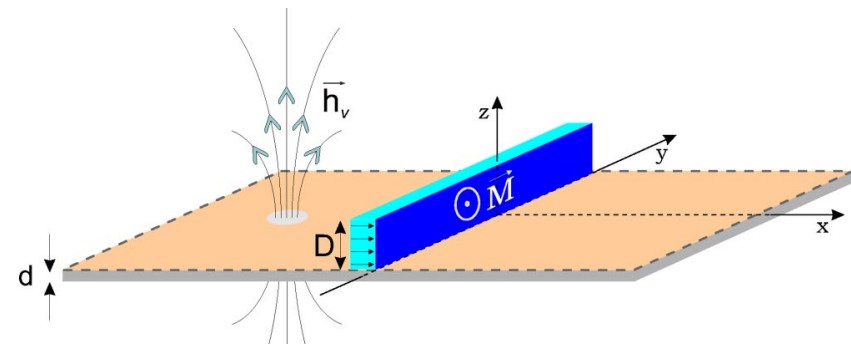

FIG. 1. (Color online) Schematic view of the system: The superconducting film underneath an in-plane magnetized stripe interacting with an external flux line.

$$
\begin{aligned}
j_{y}^{m d}(x, y, z)= & \frac{c m \Phi_{0}}{2 \pi \lambda^{3}} \frac{1}{R_{m}^{2}} \int_{0}^{\infty} d q \exp \left\{-q\left(\left|z_{m}\right|-\frac{d}{2}\right)\right\} q^{2} \\
& \times\left(\left(x_{m}^{\prime 2}-y_{m}^{\prime 2}\right) \frac{J_{1}\left(q R_{m}\right)}{q R_{m}}\right. \\
& \left.-x_{m}^{\prime 2} J_{0}\left(q R_{m}\right)\right) C(q, z)
\end{aligned}
$$

with

$$
C(q, z)=\frac{k \cosh \left[k\left(\frac{d}{2}+z\right)\right]+q \sinh \left[\left(\frac{d}{2}+z\right)\right]}{\left(k^{2}+q^{2}\right) \sinh (k d)+2 k q \cosh (k d)},
$$

where $\quad k=\sqrt{1+q^{2}}, \quad x_{m}^{\prime}=x-x_{m}, \quad y_{m}^{\prime}=y-y_{m}, \quad R_{m}$ $=\sqrt{\left(x-x_{m}\right)^{2}+\left(y-y_{m}\right)^{2}}$ is the distance between the dipole and the point of interest, and $J_{v}(\alpha)$ is the Bessel function. The coordinates $\left(x_{m}, y_{m}, z_{m}\right)$ denote the position of the dipole. The magnetic moment of the magnet is measured in units of $m_{0}=\Phi_{0} \lambda$, and all distances are scaled in units of $\lambda$. These units will be used in the rest of the paper.

To find the supercurrent induced by a finite-size FM situated above the superconductor, we make use of the superposition principle and consequently the above expressions (4) and (5) have to be integrated over the volume of the ferromagnet. Thus, the value of the current is given by ( $\alpha$ $=x, y)$

$$
j_{\alpha}(x, y, z)=\int j_{\alpha}^{m d}(x, y, z) d V^{(f m)} .
$$

\section{MAGNETIC STRIPE-VORTEX INTERACTION}

In this section, we investigate the interaction between a vortex in an infinite type-II superconducting film with thickness $d(-d / 2<z<d / 2)$ and a thin magnetic stripe with height $D$ with in-plane magnetization, i.e., $\vec{M}=\delta(x) \Theta\left(z_{1}\right.$ $-z) \Theta\left(z-z_{0}\right) M \vec{e}_{x}$ located at distance $l$ above the SC $\left(z_{0}\right.$ $\left.=l+d / 2, z_{1}=z_{0}+D\right)$. $M$ denotes the magnetic moment per unit of surface. The magnetic stripe actually represents a finite single plane of in-plane dipoles arranged perpendicular to the SC (see Fig. 1).

Inserting the well-known expression for the magnetic field of a vortex outside the SC (Refs. 14 and 15) into Eq. (2) 
we find the expression for the magnetic stripe-vortex interaction,

$$
F_{m v}=\frac{M L_{v} \Phi_{0}^{2}}{\pi \lambda} U_{m v}\left(x_{v}\right),
$$

where $x=x_{v}$ denotes the position of the vortex with vorticity $L_{v}$ with respect to the infinitely thin magnetic stripe and

$$
U_{m v}\left(x_{v}\right)=\int_{0}^{\infty} \frac{d q}{q Q} \sin \left(q x_{v}\right) \exp (-q l)[1-\exp (-q D)],
$$

with $Q=k[k+q \operatorname{coth}(k d / 2)]$. In the case of a thin superconductor $(d<\lambda)$, Eq. (7b) can be rewritten as

$$
U_{m v}\left(x_{v}\right)=f_{-}(l)-f_{+}(l)-f_{-}(l+D)+f_{+}(l+D),
$$

where

$$
\begin{aligned}
f_{ \pm}(x)= & \frac{i}{2}\left\{\ln \left(x \pm i x_{v}\right)+\Gamma\left[0,\left(x \pm i x_{v}\right) d / 2\right]\right. \\
& \left.\times \exp \left[\left(x \pm i x_{v}\right) d / 2\right]\right\}
\end{aligned}
$$

$i$ is the imaginary unit and $\Gamma(a, x)$ denotes the incomplete Gamma function. Although complex, the latter expression is easier to handle in numerical calculations [one can easily show that Eq. (8a) is a real quantity].

For an extremely thin $\mathrm{SC}(d \ll \lambda)$, we obtained

$$
f_{-}(x)-f_{+}(x)=-\frac{d}{2}\left(x \arctan \frac{x_{v}}{x}+\frac{x_{v}}{2} \ln \left(x^{2}+x_{v}^{2}\right)\right) .
$$

It is well known ${ }^{13}$ that an in-plane magnetized dipole pins the vortex at its negative pole, where the magnetic field of the vortex is parallel to the one of the dipole. Due to the dual behavior of the interaction, an antivortex present in the system would be pinned on the opposite pole. In our case of an in-plane magnetized stripe, a similar qualitative behavior is found. However, in this case, due to the fact that the stripe stretches entirely over the SC, the interaction energy depends only on the distance from the stripe, and eventually the equilibrium position of the vortex becomes an "equilibrium channel" parallel to the stripe at distance $x_{v}^{*}$. The amplitude of the interaction energy as well as the position of its extremes strongly depends on the parameters. In Fig. 2 we show the influence of the thickness of the SC film on the vortex-FM interaction (the results for $x_{v}>0$ are given, for $x_{v}<0$ the function is antisymmetric). The vortex is repelled by the positive pole of the magnet $\left(x_{v}>0\right)$ and attracted on the opposite side. One should notice that for thicker films (and fixed $l$ ), the stripe-vortex interaction is stronger and the equilibrium channel moves closer to the stripe due to the enhanced pinning.

A different interaction behavior is observed if the magnetic stripe is displaced further above the superconductor (i.e., increasing $l$ ). As one can see in Fig. 3, as the magnet is positioned higher above the SC, the interaction weakens and the equilibrium moves further away from the stripe. The physical mechanism in this case is different, and the energetically favorable position of the vortex is now determined

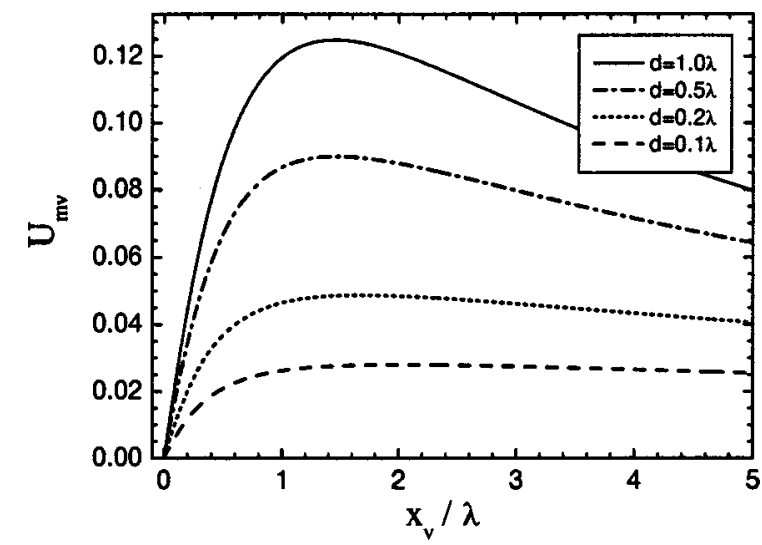

FIG. 2. The magnetic stripe-vortex interaction for different values of the thickness of the superconductor $(l=0.1 \lambda, D=0.5 \lambda)$.

by the maximum of the FM magnetic field in the SC plane. If the magnet is placed higher above the SC, the position of that maximum moves further from the stripe, and therefore the equilibrium position for the vortex shifts as well. One more parameter that influences the interaction is the height of the stripe ("wall"). Since in our calculation the magnetization is fixed $\left(M=M_{0}\right.$, the energy rescales with $\left.M\right)$ as is usually the case in the experiment, changing $D$ in our calculation increases the total magnetic moment of our magnetic structure. From this point of view it is clear that the magnetvortex interaction energy increases if the stripe is made higher (see Fig. 4). Similar to the case in Fig. 3, the preferable position of the vortex is located further away from the wall due to the shift of the maximum applied field along the $x$ direction. However, from different curves in Fig. 4 one should notice that the position of the equilibrium channel $x_{v}^{*}$ depends almost linearly on the height of the wall $D$. The results of the numerical calculation of the equilibrium position of the vortex with respect to the magnetic wall are given in Fig. 5. Independent of the thickness of the SC film, $x_{v}^{*}(D)$ becomes linear in the limit of large $D$ (i.e., for higher magnetic walls). Another interesting fact following from Fig. 5 is that for smaller heights of the wall, a crossing of the curves, obtained for different SC thickness, can be seen. This actu-

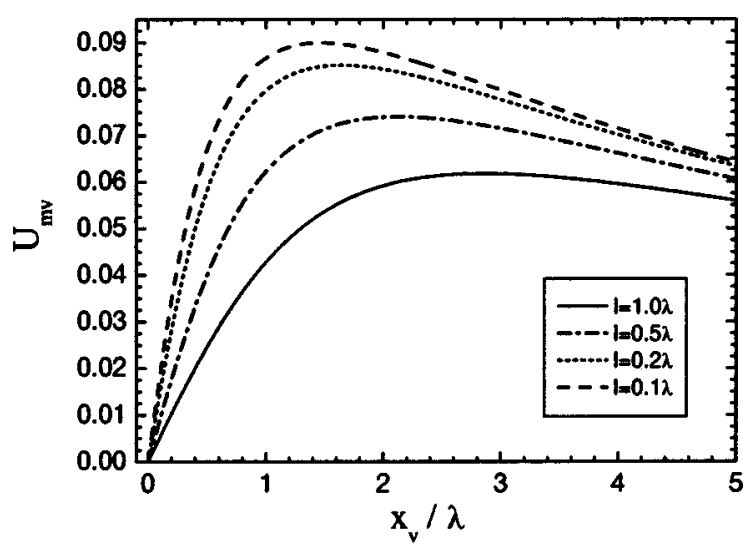

FIG. 3. The magnetic stripe-vortex interaction for different values of the distance $l$ between the stripe and the superconductor $(d$ $=0.5 \lambda, D=0.5 \lambda$ ). 


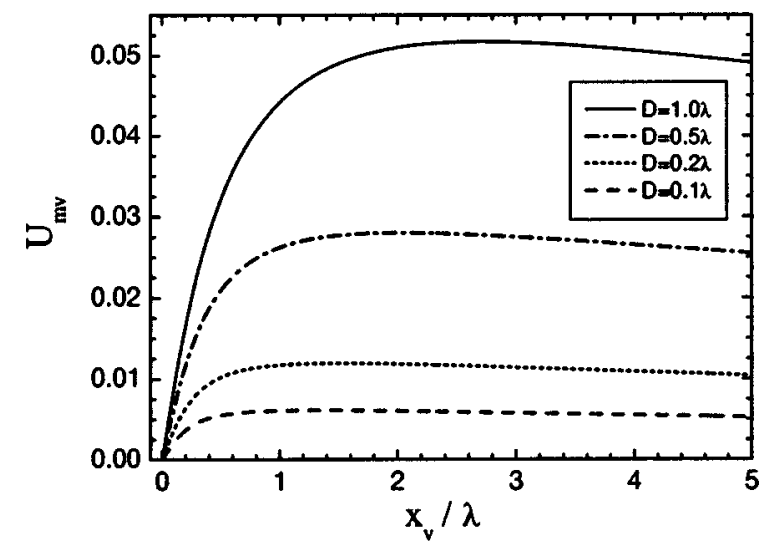

FIG. 4. The magnetic stripe- ("wall-") vortex interaction: dependence of the interaction energy on the height of the magnetic stripe $D(d=0.1 \lambda, l=0.1 \lambda)$.

ally means that for smaller $D$ the pinning site goes further away from the magnet as the thickness of the superconductor is increased, which is opposite to our analysis of Fig. 2. The reason is that with decreased $D$ the total magnetic moment (and field) decreases as well and the superconductor is able to "push" the magnetic field further from the source. As one notices in Fig. 2, and which is made more clear in the inset of Fig. 5, if the thickness of the SC increases, the vortex is attracted closer to the wall as long as the SC thickness is smaller than $\lambda$. When the thickness of the SC film exceeds the penetration depth, it becomes harder for the magnetic field to penetrate the superconductor which shifts the pinning site further away from the magnetic wall. However, after a certain SC film thickness $d$, for fixed $D$, a saturation is reached and the equilibrium vortex position remains the same, independent of the thickness of the superconducting film.

To conclude, a magnetic stripe placed on top of a superconducting film clearly separates the superconductor into two regions: (i) $x_{v}>0$ (for the orientation of the magnetization from Fig. 1) where external antivortices are attracted and



FIG. 5. The equilibrium position of the vortex when interacting with a magnetic "wall" as a function of the height of the wall for different values of the thickness of the superconductor $(l=0.1 \lambda)$. The inset shows the equilibrium vortex position as a function of the thickness of the SC. (ii) $x_{v}<0$, where additional vortex flux lines are pinned. Therefore, for the case of a superconductor in an external magnetic field, the use of a magnetic stripe (wall) is a powerful tool to preserve a vortex-free superconducting area [(i) or (ii), depending on the polarity of the applied field] in the neighborhood of the FM. Although our calculations were done for the case of an infinite SC, the same conclusions can apply to finite-size superconductors. Moreover, depending on the parameters (as we have shown in Figs. 2-5), the position of the pinning site can actually be quite far from the stripe, and the (anti)vortices can actually be expelled completely from the superconductor. Knowing that moving free vortex lines are responsible for the energy dissipation and lowering of the critical parameters in superconducting systems, our results could play a significant role in applied superconductivity.

So far, our calculations were done independently of the temperature, since all quantities in our analysis were scaled by units in which all the temperature-dependent parameters were incorporated. Therefore, in this section, we include the temperature indirectly, through $\lambda$, whose temperature dependence is given by

$$
\lambda(T)=\frac{\lambda(0)}{\sqrt{\left|1-T / T_{c 0}\right|}},
$$

where $\lambda(0)$ denotes the penetration depth at zero temperature and $T_{c 0}$ is the critical temperature at zero magnetic field.

We repeat the calculations of the magnetic stripe-vortex interaction energy [Eq. (7b)], with a difference that now all the distances are expressed in units of $\lambda(0)$ instead of $\lambda$ and the unit of magnetization becomes $M_{0}=\Phi_{0} / \lambda(0)^{2}$. Equation (7b) now becomes

$$
\begin{gathered}
F_{m v}=\frac{M L_{v} \Phi_{0}^{2}}{\pi \lambda(0)} U_{m v}\left(x_{v}\right), \\
U_{m v}\left(x_{v}\right)=\int_{0}^{\infty} \frac{d q T_{f}}{q k\left[k+q \operatorname{coth}\left(k d T_{f} / 2\right)\right]} \sin \left(q x_{v} T_{f}\right) \\
\times \exp \left(-q l T_{f}\right)\left[1-\exp \left(-q D T_{f}\right)\right],
\end{gathered}
$$

where $T_{f}=\sqrt{\left|1-T / T_{c 0}\right|}$. The results of this calculation are given in Fig. 6 for three different temperatures $T / T_{c}$ $=0.1,0.5$, and 0.8 . Obviously, as we approach the critical temperature, the interaction between the vortex and the magnetic stripe becomes weaker [see Fig. 6(a)]. Analogously, the pinning weakens, and the equilibrium position of the vortex in this case moves further from the magnet [open dots in Fig. 6(a)]. In Fig. 6(b) we show the dependence of the energetically favorable position of the vortex as a function of the temperature. One should notice the abrupt increase of the magnet-vortex equilibrium distance when we approach the critical temperature. Therefore, at temperatures close to the superconducting/normal state transition the magnetic stripe can effectively protect the neighboring superconductor from vortices since they are pinned far from the stripe. 

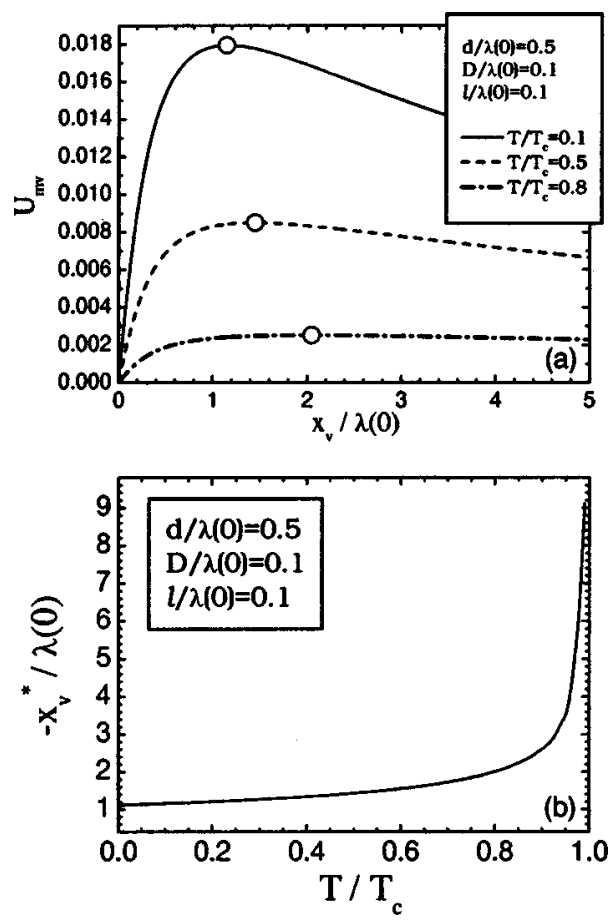

FIG. 6. (a) The magnetic stripe-vortex interaction energy (open dots denote the extremes in the energy) and (b) the equilibrium position of the vortex, as a function of the temperature of the system.

\section{FIELD POLARITY DEPENDENT VORTEX PINNING BY AN IN-PLANE MAGNETIZED BAR}

In this section, we analyze the pinning properties of a magnetic bar, magnetized along its longer side (see Fig. 7 for definition of the variables). Using Eq. (2) and the wellknown expressions for the magnetic field of the vortex, we obtain the pinning potential as

$$
F_{m v}=\frac{M L_{v} \Phi_{0}^{2}}{2 \pi \lambda} U_{m v},
$$

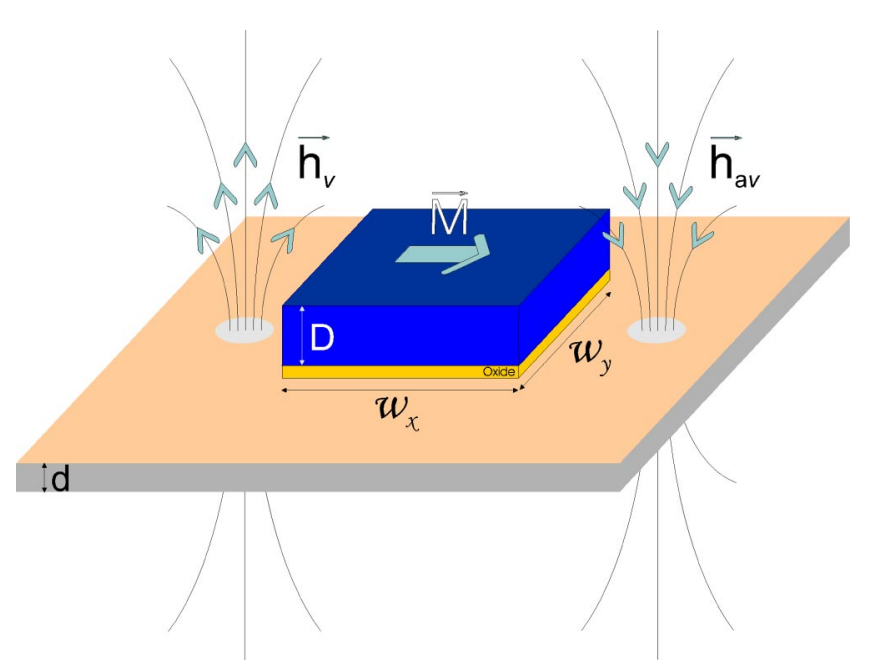

FIG. 7. (Color online) The superconducting film with in-plane magnetized bar on top of it (separated by an oxide layer) interacting with a(n) (anti)vortex: an oblique view of the system.
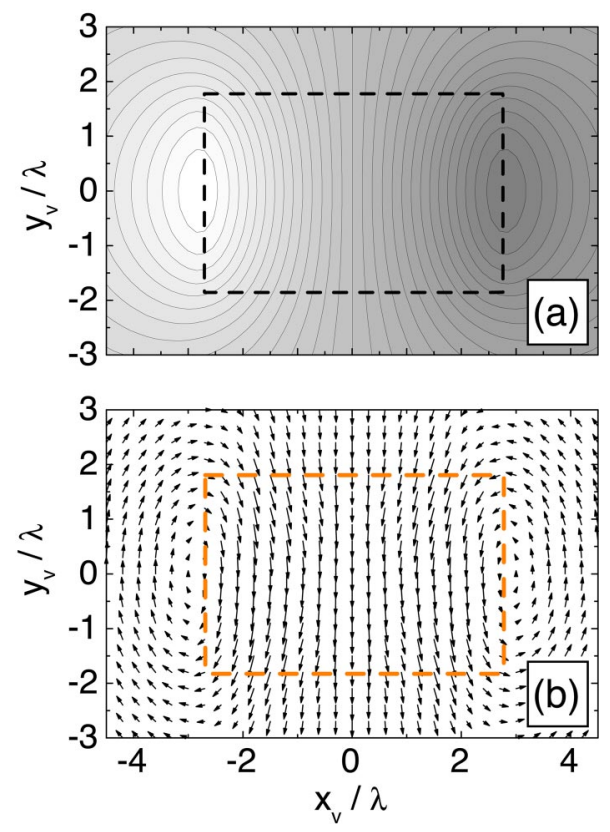

FIG. 8. (Color online) (a) The contour plot of the magnetic bar-vortex interaction energy [white (dark) color-low (high) energy] and (b) the vector plot of the Meissner current induced in the $\mathrm{SC}$ due to the presence of the magnetic bar. The dashed line indicates the edges of the magnet. The parameters used are $w_{x}=5.4 \lambda$, $w_{y}=3.6 \lambda, d=0.5 \lambda, D=0.35 \lambda$, and thickness of the oxide $l$ $=0.2 \lambda$.

$$
\begin{aligned}
U_{m v}= & \int_{0}^{\infty} \frac{d q}{q Q} \int_{-w_{y} / 2}^{w_{y} / 2} d y\left[J_{0}\left(q R^{+}\right)-J_{0}\left(q R^{-}\right)\right] \exp (-q l) \\
& \times[\exp (-q D)-1],
\end{aligned}
$$

where $M$ denotes the magnetization of the bar, $D$ its thickness, and $R^{ \pm}=\sqrt{\left(y-y_{v}\right)^{2}+\left(w_{x} / 2 \pm x_{v}\right)^{2}}$. $\left(x_{v}, y_{v}\right)$ gives the position of the vortex with vorticity $L_{v}$ where the coordinate center is located under the center of the magnet.

As one can see in Fig. 8, where the contour plot of the interaction energy is shown, the vortex is attracted to the negative pole of the magnetic bar where the magnetic field is parallel to the field of the vortex (and repelled in the opposite case), which is similar to the case of the out-of-plane magnetized dipole (magnetic moment parallel to the external flux lines attracts the vortex, and vice versa). This conclusion also follows from the observation of the interaction between the induced currents and the vortex. The vector plot of the Meissner current, calculated using Eq. (6), shows [see Fig. $8(\mathrm{~b})]$ that the current has an antivortexlike direction at the position where the magnetic field of a magnetic bar is parallel to the vortex magnetic field (negative pole) and a vortexlike direction on the positive pole. Note that these are the Meissner currents which oppose to the field of the magnetic bar and do not correspond to the vortex-antivortex pair.

Obviously, in the case of in-plane magnetization, the vortex will be repelled at the pole of the magnetic bar to which the magnetic moment points and pinned on the opposite side (and vice versa for the antivortex). Thus, there is a fieldpolarity-dependent vortex pinning. However, the pinning position of the (anti)vortex depends not only on the direction of 
the external flux lines but also on the value of the magnetization of the bar as well; namely, if the magnetization of the bar is strong enough to create a vortex-antivortex pair itself, the pinning properties of the system change. Such a case was recently experimentally investigated by Van Bael et al., ${ }^{11}$ where it was found that the stray field of the in-plane magnetic dipole induces a vortex-antivortex pair at the poles, at positions predicted by our analysis (vortex towards negative pole, antivortex towards positive pole). Therefore, this asymmetric pinning potential provides stability for vortexantivortex configurations. In order to compare our results with the experimental ones, we performed our analysis with the value of the parameters given in Ref. 11 (renormalized to our units): $w_{x}=5.4 \lambda, w_{y}=3.6 \lambda, d=0.5 \lambda, D=0.35 \lambda, l$ $=0.2 \lambda$, and $\kappa \approx 1$.

First, we determine the criterion for the nucleation of a vortex-antivortex pair. In order to obtain a threshold value of magnetization for which a state with a vortex-antivortex pair is more energetically favorable than the Meissner state, we compared the total energy of the system in these two cases. The contribution to the energy of the system due to the appearance of such a pair equals

$$
\Delta F=F_{m v}+F_{\text {mav }}+F_{\text {vav }}+F_{s v}+F_{s a v},
$$

where $F_{v a v}$ and $F_{s v}$ denote the vortex-antivortex interaction energy and the self-energy of the vortex, respectively. These energies are obtained from ${ }^{15}$

$$
\begin{gathered}
F_{v a v}=-\frac{L_{v} L_{a v} \Phi_{0}^{2}}{8 \pi^{2} \lambda} U_{v a v}, \\
U_{v a v}=d K_{0}(R)+2 \int_{0}^{\infty} d q \frac{J_{0}(q R)}{k^{2} Q},
\end{gathered}
$$

where $R=\sqrt{\left(x_{v}-x_{a v}\right)^{2}+\left(y_{v}-y_{a v}\right)^{2}}$ is the distance between the vortex and the antivortex, $K_{0}(x)$ is the MacDonald function, and $L_{v}, L_{a v}$ are the vorticity of the vortex and antivortex, respectively, and

$$
\begin{gathered}
F_{s v}=\frac{L_{v}^{2} \Phi_{0}^{2}}{16 \pi^{2} \lambda} U_{s v}, \\
U_{s v}=d \ln \kappa+2 \tanh \left(\frac{d}{2}\right) \ln \left(1+\operatorname{coth} \frac{d}{2}\right) .
\end{gathered}
$$

From the condition $\Delta F=0$ the value of the FM magnetization necessary for the appearance of the vortex-antivortex pair can be estimated as

$$
M^{*}=\frac{1}{8 \pi} \frac{U_{s v}-U_{v a v}}{U_{m v}} .
$$

In the more general case, the transition from $L_{v}=L_{a v}=L$ to the $L_{v}=L_{a v}=L+1$ state is determined by the FM magnetization

$$
M_{L \rightarrow L+1}^{*}=\frac{(2 L+1)}{8 \pi} \frac{U_{s v}-U_{v a v}}{U_{m v}}
$$



FIG. 9. (Color online) The threshold magnetization of the magnetic bar necessary for the nucleation of the first and second vortexantivortex pair (solid lines) as a function of the geometrical ratio (between the sides) of the bar (for fixed volume of the magnet, where $S_{x y}=w_{x} w_{y}$, with parameters taken from Ref. 11). The dashed lines denote the changing of the vortex pinning properties of the bar: in the shaded (white) regions, an external flux line is pinned at the positive (negative) pole of the FM.

In order to use Eqs. (16) and (17), one should first determine the positions of the vortex and the antivortex. This can easily be done if the total interaction energy in the system is calculated [Eq. (13), without the self-energy terms] with the position of the vortex $x_{v}$ as a free parameter $\left(x_{a v}=-x_{v}, y_{v}\right.$ $=y_{a v}=0$ ). The minimal interaction energy then determines the exact equilibrium position of the vortex-antivortex pair.

Using Eq. (16) for the parameters taken from the experiment of Van Bael et al. ${ }^{11}$ we obtain $M^{*} \approx 0.08 M_{0}$. Unit $M_{0}$ in the SI system equals $M_{0}=4 \pi \Phi_{0} \lambda / \mu_{0} \approx 2.07 \times 10^{6} \mathrm{~A} / \mathrm{m}$ $(\lambda \approx 100 \mathrm{~nm})$. Knowing that the typical magnetization of $\mathrm{Co} / \mathrm{Pt}$ dots used in the experiments equals $\approx 2.5 \times 10^{5} \mathrm{~A} / \mathrm{m}$, one can see that the $M / M_{0}$ ratio equals 0.12 and our analysis shows that such a magnetic bar can create exactly one vortex-antivortex pair, which corresponds to the experimental findings of Ref. 11. It is important to emphasize that the critical magnetization of the bar to induce a vortexantivortex pair depends strongly on the geometrical parameters of the bar. To investigate this, we repeated our analysis as a function of the ratio between the sides of the bar, but keeping the volume of the magnet $\left(V_{f m}=S_{x y} D\right)$ and other parameters the same (magnetization is directed along the $x$ axis). The results shown in Fig. 9 suggest that the magnetic bar is more magnetically effective for pinning at its poles if it is magnetized along its longer side. However, the threshold magnetization for the nucleation of vortices decreases monotonously as the "magnetization side" of the magnetic bar 




FIG. 10. (Color online) The trajectory of the external flux line when interacting with a magnetic bar and its induced vortexantivortex pair (colored dots) for different values of the magnetization of the bar (the remaining parameters are taken from Ref. 11).

is made shorter (see Fig. 9). However, the flux penetrating the superconductor approximately remains the same along the solid lines in Fig. 9. Using the magnetostatic calculation, we obtained the critical condition for the nucleation of the vortex-antivortex pairs as $\Phi^{+} / \Phi_{0}=\nu\left(L_{v}+1 / 2\right)$, where $\Phi^{+}$ denotes the flux through the region of the positive stray field of the magnet at the SC surface, and $\nu=1.073,1.064,1.054$, for $w_{y} / w_{x}=1,2,3$. Using the values of the parameters from Ref. $11\left(w_{y} / w_{x}=0.667\right)$, we obtain $\nu=1.094$. Therefore, for the first stable vortex-antivortex pair a lower value of flux is needed, namely, $\Phi^{+} / \Phi_{0}=0.547$, while the appearance of additional pairs is quantized in $\Delta \Phi^{+} / \Phi_{0}=1.094$. The smaller value of the threshold flux in the first case can be explained by the contribution of the local currents which effectively add an amount of flux to create a fluxoid of exactly $L_{v} \Phi_{0}$ at the pole if the stray field of both poles of the magnetic bar creates a (positive or negative) flux between $\left(L_{v}-1 / 2\right) \Phi_{0}$ and $L_{v} \Phi_{0}$. Note that this is similar to the superconducting ring case ${ }^{16}$ where the $L \rightarrow L+1$ ground vortex state transitions take place for $\Phi \approx(L+1 / 2) \Phi_{0}$ where the approximate sign becomes equality for large radius thin rings.

As we have shown before (see Fig. 8), if no vortexantivortex pair is induced in the SC by the magnetic bar, an additional positive flux line (vortex) will be pinned on the negative pole of the bar (i.e., $x_{v}<0$ ). Now, we assume that the value of the magnetization of the bar is such that one vortex-antivortex pair appears on the poles of the bar (vortex at the negative pole and antivortex at the positive pole, for $0.08<M / M_{0}<0.24$, for $w_{y} / w_{x}=0.667$ ) and we add an extra vortex in the system. The total force acting on this vortex consists of the vortex-magnetic bar, and vortex-vortex and vortex-antivortex interactions. To investigate the interaction of a magnetic bar and its vortex-antivortex pair with an additional external flux line, we put the vortex in different positions (open dot in Fig. 10) and follow its trajectory using MD simulations. In our quasistatic case, if the vortex moves, the FM-vortex interaction force is opposed by viscous forces of the form $-\eta \cdot v_{v}$, where $\eta$ is the viscosity coefficient and $v_{v}$ the velocity of the vortex. In equilibrium, these forces are equal and the motion of the vortex can be analyzed. Since we were not interested in real-time simulations, we assumed $\eta$ $=1$. The components of the force were obtained by simple derivation of the expressions for the contributions to the total interaction energy, which were given before in this section. The results of this MD simulation are shown in Fig. 10 for different values of the magnetization $M$. We choose the initial position of the vortex at the same distance from the poles of the magnet, in order not to favor any of the possible pinning sites. Obviously, for smaller magnetization of the bar (just enough to create a vortex-antivortex pair at the poles), the external flux line is attracted by the antivortex and pinned on the positive pole of the bar, which is opposite to the situation for $M / M_{0}<0.08$ shown in Fig. 8. This is also illustrated in Fig. 11(a) where we show a contour plot of the spatial variation of the total energy on the position of the external vortex. However, the magnet-vortex interaction on the positive pole of the bar is still repulsive, and for larger magnetization $\left(M / M_{0}=0.12\right)$ the vortex is attracted to the antivortex (where annihilation occurs), but this trajectory towards the equilibrium position is distorted, since the magnetic bar is trying to pin the vortex at its negative pole.

Eventually, for further increased magnetization (and still smaller than $M_{1 \rightarrow 2}^{*}$ ) the magnet-vortex interaction overwhelms the vortex-antivortex attraction, and the vortex is pinned at the negative pole of the magnet, although a magnet-induced vortex is already present there. The shape of the most energetically favorable vortex state at the negative pole of the magnet (multivortex or giant vortex) depends on the parameters, mainly on the GL parameter $\kappa$, which determines the vortex-vortex interaction and the self-energy of vortices. As one can see in Fig. 11(b), the external vortex in this case is pinned next to the vortex induced by the FM dipole. In this calculation the position of the FM-induced vortex-antivortex pair is kept fixed when the external vortex is moved over the SC surface. The critical magnetization at which the pinning switches from the positive to the negative pole of the FM is shown by the thick dashed curve in Fig. 9. The physical reason for the switching of the pinning position is the reversal of the direction of the screening currents near the vortex and the antivortex. This is illustrated in Figs. 12(b)-(d). In Fig. 12(a) we show the interaction energy of the external vortex with the FM and the induced vortexantivortex pair, along the $y_{v}=-x_{v} / 2$ direction [which is approximately along the line where energy minimum and maximum are found in Fig. 11(b)] for different values of the magnetization of the FM. The change of the pinning behavior of the FM bar is clearly visible. In Figs. 12(b)-(d) the corresponding vector plots of the screening currents are shown. Obviously, with increasing magnetization of the magnetic bar, the current flow in the SC changes. At the negative pole of the FM, for lower magnetization [Fig. 12(b)], the current is vortexlike, due to the presence of an induced vortex there. However, as we increase the magnetization [Fig. 12(c)], an antivortex type of current flow appears close to the vortex site, resulting ultimately in a pure anti- 




FIG. 11. (Color online) Contour plot of the interaction energy of an external flux line with the screening currents induced by the FM in case that the FM bar induces a vortex-antivortex pair [white (dark) color-low (high) energy] for the magnetization of the magnetic bar (a) $M / M_{0}=0.12$ and (b) $M / M_{0}=0.20$. The position of the FM-induced vortex-antivortex pair is taken fixed.

vortexlike current at the negative pole of the magnet as the magnetization approaches the critical value for the nucleation of the next vortex-antivortex pair [Fig. 12(d)]. In all cases, the stable position of an external vortex is determined by the position of this quasi-antivortex, which explains the switching of the pinning behavior with increasing magnetization of the magnetic bar.

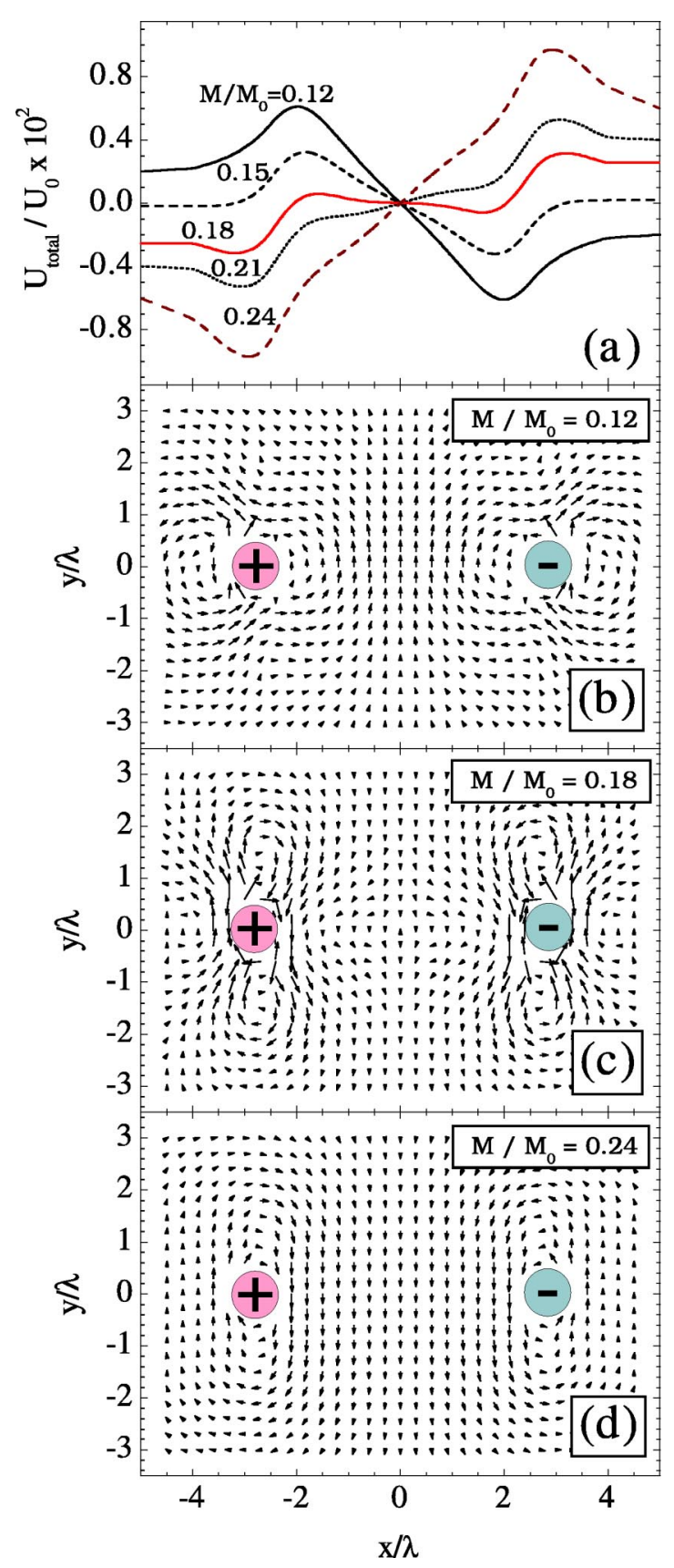

FIG. 12. (Color online) (a) The interaction energy (in units of $\left.U_{0}=\Phi_{0}^{2} / \pi \lambda\right)$ of an external flux line with the FM and induced vortex-antivortex pair along the $y_{v}=-x_{v} / 2$ direction [see Fig. 11(b)] for different values of the magnetization of the magnetic bar. (b)-(d) Vector plots of the screening currents in the SC due to the presence of the FM and the induced vortex-antivortex pair.

To summarize, in Ref. 11 it was found experimentally that in the case of vortex pinning by magnetic bars with in-plane magnetization such that one vortex-antivortex pair is induced in the superconductor, an extra external vortex is attracted by the antivortex (and vice versa, for external antivortex) and they annihilate each other. The authors refer to this phenomenon as a field-polarity-dependent pinning. Our simulation shows that for the given experimental parameters this is indeed the case. But, our analysis shows that this phenomenon 

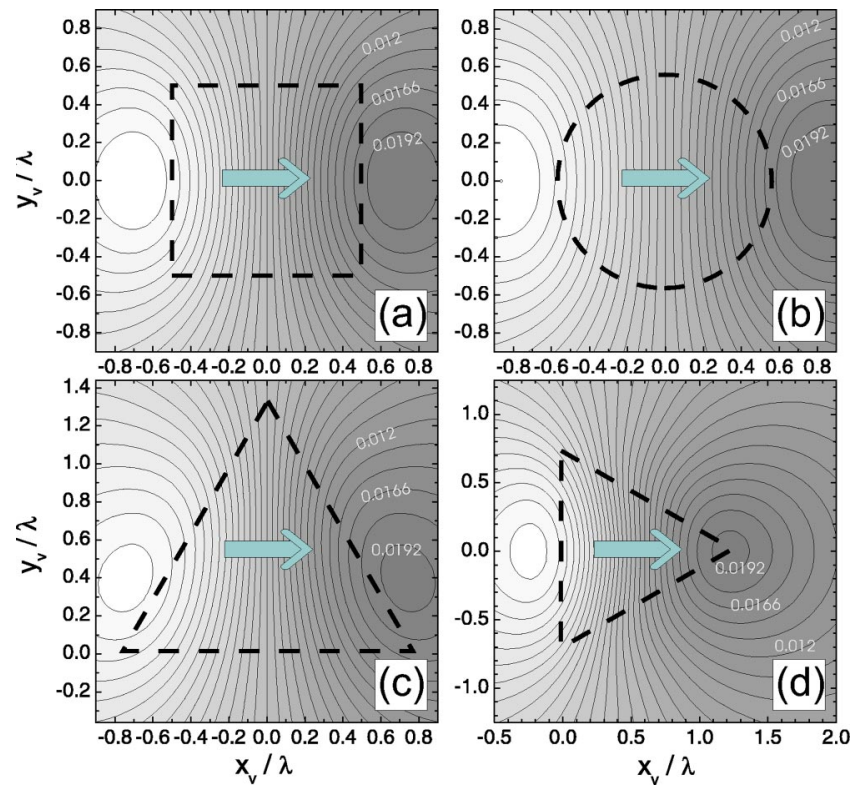

FIG. 13. (Color online) The contour plots of the in-plane magnet-vortex interaction energy for different shapes of the magnet. Dashed lines indicate the edges of the magnetic structures $(d$ $=0.25 \lambda, D=0.25 \lambda, l=0.1 \lambda, M=M_{0}$, surface of the magnet base is the same for all structures $S=\lambda^{2}$ ). All plots are given with the same energy scale (some of the values of the contour lines are indicated).

not only depends on the polarity of the external magnetic field but also on the strength of the magnetization of the magnetic bar. For larger magnetization of the magnet, we found that the external vortex can be pinned on the opposite side, i.e., at the negative pole of the bar, next to the magnetinduced vortex (as shown in Figs. 10-12). This remarkable phenomenon should be easily observable using the same experimental procedure as in Ref. 11, if samples with micromagnets with a larger magnetization are used. In that case, one should avoid the annihilation between the external flux line and the antivortex created by the neighboring dipole, and make the magnetic pinning lattice sparser.

\section{INFLUENCE OF CORNERS/DEFECTS IN THE MAGNET GEOMETRY ON THE INTERACTION ENERGY}

In this section, we investigate the influence of the magnet geometry on the pinning of vortices in the case when no vortex-antivortex pair is nucleated. For magnets with out-ofplane magnetization ${ }^{14}$ it was found that the qualitative behavior of the magnet-vortex interaction does not depend on the geometry, as was found by comparing the results for disk, square, and triangular FM's. However, a difference in the dynamics of pinning was noted, since the vortex approaches its equilibrium position under the magnet following the trajectory over the corners of the magnet, rather than perpendicular to the sides, in the case of square and triangular FM's. Also, by introducing hole(s) in the magnetic structure, it was shown how one can create different local minima in the interaction energy and manipulate the equilibrium position of the vortices.
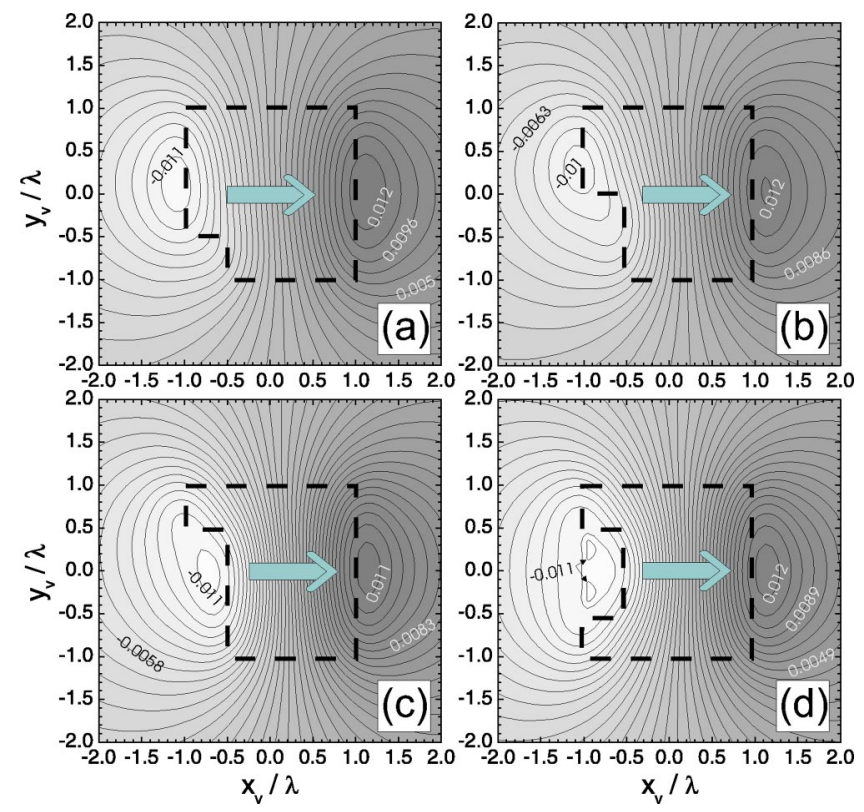

FIG. 14. (Color online) The contour plots of the in-plane magnet-vortex interaction energy for different shapes of the magnet. Dashed lines illustrate the edges of the magnetic structures $(d$ $\left.=0.25 \lambda, D=0.25 \lambda, l=0.1 \lambda, M=M_{0}\right)$. The plots are given with slightly different energy scales (some of the prominent contour lines are labeled).

We repeated this analysis for in-plane magnetization of the magnet, for the magnetic disk, square, and triangle with fixed thickness and volume. We use Eq. (12b) to calculate numerically the magnet-vortex interaction energy profile, where in the integration over the magnet boundaries $\left( \pm w_{x} / 2\right)$ we substitute the functions describing the magnet's shape. The results are shown in Fig. 13. The qualitative behavior of the vortex-magnet interaction is the same in all cases. Moreover, the amplitudes of the interaction potential appear to be very similar (all the contour plots in Fig. 13 are given with the same energy scale). One can see that in the case of symmetric magnets with respect to the magnetization direction the contour lines follow the shape of the magnet and the extreme interaction energy regions are set parallel to the opposite sides of the magnet. However, if the symmetry is broken [see Fig. 13(d) for the case of a triangular magnet with magnetization perpendicular to one of its sides] the interaction symmetry is broken and the amplitude of the energy is somewhat higher at the negative pole of the magnet.

Next we introduced an asymmetry in the geometry of the magnet, not only by rotating the magnetization in the $x y$ plane but also by introducing edge defects. Referring to our previous article, ${ }^{14}$ we may model the removed area as a superposition of two ferromagnets with opposite magnetization. The smaller size magnet with the opposite magnetization can model the hole (defect) in the larger magnetic structure. As one can see in Fig. 14, the equilibrium position of the vortex can be nicely manipulated by an edge defect on the side of the magnet. When a defect is located in the corner of the magnetic square [see Fig. 14(a)], the equilibrium position for the vortex shifts slightly away from the defect, and the symmetry of the positions of extremes in the interaction energy is broken. As we spread the defect further along the 
negative pole of the magnet, the energetically favorable position of the vortex moves further up, i.e., it "runs" away from the upper edge of the defect [Fig. 14(b)]. However, when the inner edge of the defect becomes longer than the outer magnet edge, the ground state for the vortex moves back towards the central position [Fig. 14(c)]. In this case, the part of the magnet that is sticking out acts as a defect and breaks the symmetry of the interaction. Therefore, to further investigate the competition of these two + and - defects, we set an edge defect in the center of the magnetic structure [Fig. 14(d)]. In this case (for given parameters), the central equilibrium vortex position breaks up into two, and the contour plot of the energy shows two equal minima close to the upper and lower edges of the defect. Thanks to this feature, a possible use of this system for quantum computing can be analyzed similarly to the quantum systems proposed before (see, for example, Ref. 17).

\section{CONCLUSION}

To summarize, we applied the London theory to investigate flux pinning in SC films due to the presence of an inplane magnetized ferromagnet situated above the SC, where the finite thickness of both FM and the SC are taken into account. In the case of a magnetic stripe on top of a superconductor, we obtained a semianalytic expression for the FM-vortex interaction energy and analyzed the dependence of the interaction potential on the system parameters. We found that the equilibrium position for an external flux line pinned by the stripe lies in the channel, parallel to the magnetic structure. The exact position of this equilibrium channel depends on both thickness of the SC and the magnet. Also, all the flux lines of the same polarity are found to be pinned on the same side of the stripe. Therefore, using such a magnetic structure on top of a superconductor in the pres-

*Electronic address: francois.peeters@ua.ac.be

${ }^{1}$ J.I. Martin, M. Velez, J. Nogues, and I.K. Schuller, Phys. Rev. Lett. 79, 1929 (1997).

${ }^{2}$ M.J. Van Bael, K. Temst, V.V. Moshchalkov, and Y. Bruynseraede, Phys. Rev. B 59, 14674 (1999).

${ }^{3}$ M. Lange, M.J. Van Bael, Y. Bruynseraede, and V.V. Moshchalkov, Phys. Rev. Lett. 90, 197006 (2003).

${ }^{4}$ L.N. Bulaevskii, E.M. Chudnovsky, and M.P. Maley, Appl. Phys. Lett. 76, 2594 (2000).

${ }^{5}$ I.K. Marmorkos, A. Matulis, and F.M. Peeters, Phys. Rev. B 53, 2677 (1996).

${ }^{6}$ Sa-Lin Cheng and H.A. Fertig, Phys. Rev. B 60, 13107 (1999).

${ }^{7}$ I.F. Lyuksyutov and V.L. Pokrovsky, Phys. Rev. Lett. 81, 2344 (1998).

${ }^{8}$ S. Erdin, A.F. Kayali, I.F. Lyuksyutov, and V.L. Pokrovsky, Phys. Rev. B 66, 014414 (2002). ence of a homogeneous magnetic field may lead to a superconducting region without vortices. In the case of finite-size superconductors, if the pinning channel is relatively far from the stripe, the whole superconducting sample could be made vortex free, which is highly important for applications.

In the case of pinning of vortices by an in-plane magnetic bar, we discussed the field-polarity-dependent pinning. We found that a vortex or antivortex in these systems is pinned on the opposite poles of the bar. However, on which pole the vortex will be pinned depends on the strength of the magnetization of the magnet and the possible presence of a vortexantivortex pair induced in the SC by the magnet itself (see Figs. 10-12). Our results agree with the experiment of Ref. 11 and give directives for future experimental considerations of similar heterostructures, since our analysis predicts that the position of the pinned vortex can be tuned by changing the strength of the magnetization of the FM.

We extended this approach further to investigate the influence of edge defects of the magnetic structure on the pinning potential and we found that such defects break the symmetry of the vortex-magnet interaction. Moreover, different local minima in the energy were found. For symmetric position of the defect with respect to the edges of the magnet, we found that two degenerate vortex states are possible which differ in the position of the vortex, making this SC-FM structure interesting as a possible qubit.

\section{ACKNOWLEDGMENTS}

This work was supported by the Flemish Science Foundation (FWO-Vl), the Belgian Inter-University Attraction Poles (IUAP), the "Onderzoeksraad van de Universiteit Antwerpen" (GOA), and the ESF program on "Vortex matter." Stimulating discussions with D. Vodolazov are gratefully acknowledged.

${ }^{9}$ L.E. Helseth, Phys. Rev. B 66, 104508 (2002); 67, 139903 (2003).

${ }^{10}$ D.J. Morgan and J.B. Ketterson, Phys. Rev. Lett. 80, 3614 (1998).

${ }^{11}$ M.J. Van Bael, J. Bekaert, K. Temst, L. Van Look, V.V. Moshchalkov, Y. Bruynseraede, G.D. Howells, A.N. Grigorenko, S.J. Bending, and G. Borghs, Phys. Rev. Lett. 86, 155 (2001).

${ }^{12}$ Y. Nozaki, Y. Otani, K. Runge, H. Miyajima, B. Pannetier, J.P. Nozières, and G. Fillion, J. Appl. Phys. 79, 8571 (1996).

${ }^{13}$ M.V. Milošević, S.V. Yampolskii, and F.M. Peeters, Phys. Rev. B 66, 174519 (2002).

${ }^{14}$ M.V. Milošević and F.M. Peeters, Phys. Rev. B 68, 094510 (2003).

${ }^{15}$ M. Fusco-Girard and F. Mancini, Physica B \& C 123, 75 (1983).

${ }^{16}$ B.J. Baelus, F.M. Peeters, and V.A. Schweigert, Phys. Rev. B 61, 9734 (2000).

${ }^{17}$ L.B. Ioffe and M.V. Feigelman, Phys. Rev. B 66, 224503 (2002). 